\title{
"Shock-Pella": Combined management of an undilatable ostial left circumflex stenosis in a complex high-risk interventional procedure patient
}

\author{
Andrea Buono ${ }^{1}$, Alfonso Ielasi ${ }^{1}$, Giuseppe De Blasio ${ }^{2}$, Maurizio Tespili ${ }^{1}$ \\ ${ }^{1}$ Interventional and Clinical Cardiology Unit, S. Ambrogio Cardio-Thoracic Center, Milan, Italy \\ ${ }^{2}$ Cardiology Unit, IRCCS Istituto Ortopedico "Galeazzi”, Milan, Italy
}

A 67-year-old woman with stage 4 chronic kidney disease, implantable cardioverter-defibrillator and a history of multiple coronary interventions, both percutaneous (stenting of anterior descending artery [LAD], ramus and right coronary artery [RCA]) and surgical left internal mammary artery [LIMA] graft on LAD and saphenous vein grafts [SVG] on ramus and RCA), was admitted because of congestive heart failure with evidence of severe left ventricular ejection fraction decrease (25\%). Coronary angiography showed occluded LIMA and SVG to ramus, patent SVG to RCA and a critical ostial left circumflex artery (LCx) stenosis (Fig. 1A). Since myocardial perfusion scintigraphy showed no viability on anterior wall and apex (Fig. 1B), a protected LCx lesion revascularization was attempted, positioning a circulatory mechanical support (Impella CP; Abiomed, Danvers, MA).
Non-compliant balloons did not fully expand during lesion predilatation, probably due to severe fibrocalcification and protruding ramus stent struts (intravascular ultrasound catheter did not cross the lesion) (Fig. 1C). Intravascular lithotripsy (IVL) was then performed (Shockwave Medical, Fremont, CA), inflating a $3.0 \times 12 \mathrm{~mm}$ balloon (at 4-6 atm for 8 cycles of 10 pulses each) with angiographic evidence of complete device expansion (Fig. 1D) and subsequent optimal lesion predilation with non-compliant balloon (Fig. 1E). A $3.5 \times 15 \mathrm{~mm}$ drug-eluting stent was successfully implanted (Fig. 1F). This is a case of complex, high-risk interventional procedure managed with combined strategy "Impella-assisted IVL" to prevent the risk of hemodynamic compromise in a time-demanding procedure where an optimal and aggressive lesion debulking was required.

Conflict of interest: None declared

Address for correspondence: Alfonso Ielasi, MD, FESC, Clinical and Interventional Cardiology Unit,

S. Ambrogio Cardio-Thoracic Center, Via Faravelli 16, 20149, Milan, Italy, tel: +39 02-33127714, fax: +39 02-33127717, e-mail: alielasi@hotmail.com; alfonso.ielasi@gmail.com

Received: 31.01.2020 Accepted: 11.04 .2020 


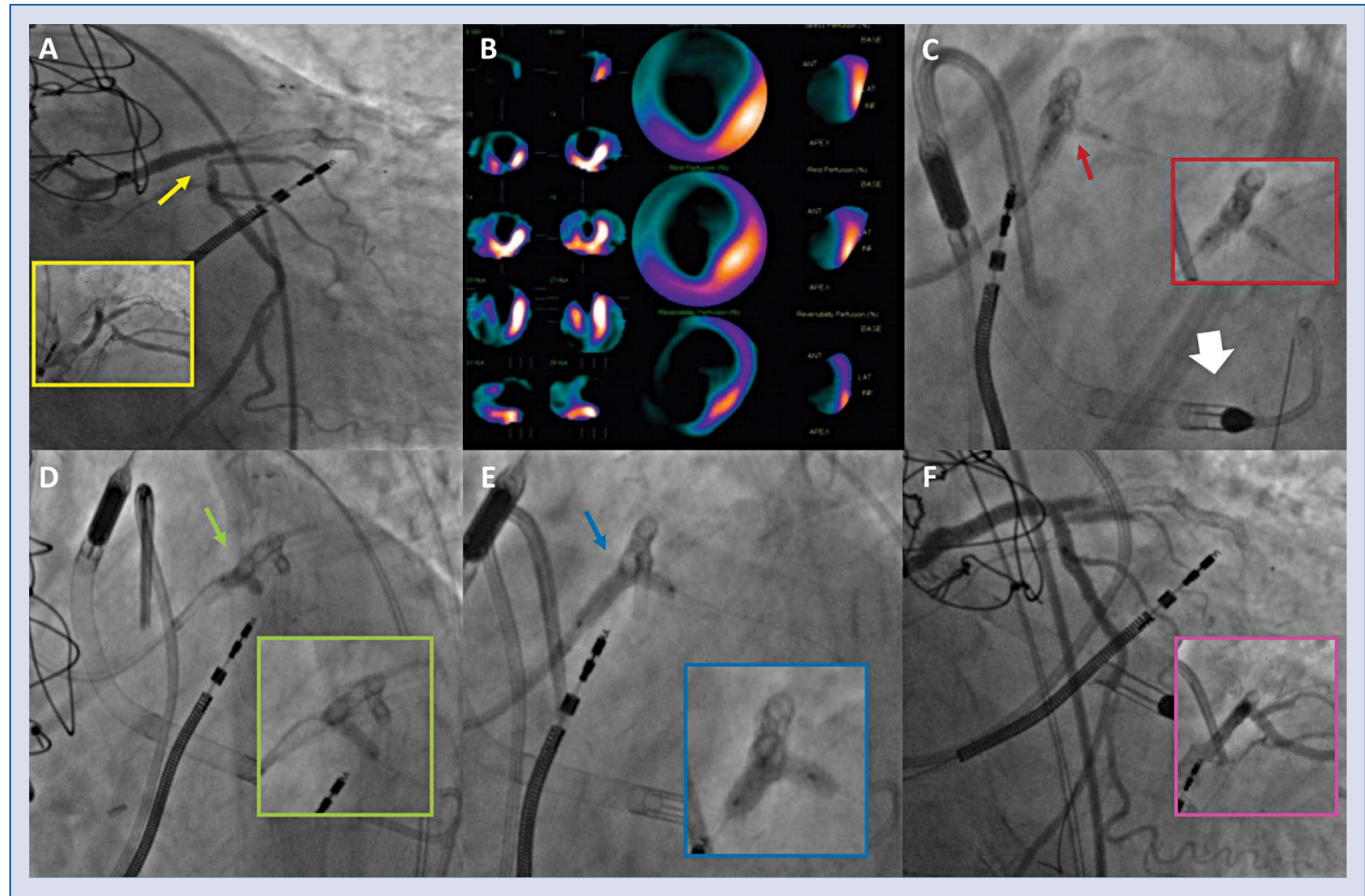

Figure 1. A. Severe ostial left circumflex lesion (yellow arrow); yellow box shows a second angiographic view confirming the critical lesion entity; B. Absence of anterior myocardial viability at myocardial perfusion scintigraphy; C. Impella CP positioned in the left ventricle across the aortic valve (withe arrow); "dog-bone" sign for an undilatable lesion by multiple non-compliant balloon inflations (red arrow and box); D. Optimal Shockwave balloon inflation (green arrow), confirmed in a second angiographic view (green box); E. Full $3.5 \times 20 \mathrm{~mm}$ non-compliant balloon expansion after intravascular lithotripsy (blue arrow and box); F. Excellent final angiographic result following ostial left circumflex lesion stenting (pink arrow and box). 